\title{
Aa. Vv., Sexualité et écriture
}

\section{Elena Pessini}

\section{OpenEdition}

\section{Journals}

\section{Édition électronique}

URL : https://journals.openedition.org/studifrancesi/39886

DOI : 10.4000/studifrancesi.39886

ISSN : 2427-5856

\section{Éditeur}

Rosenberg \& Sellier

\section{Édition imprimée}

Date de publication : 1 décembre 2004

Pagination : 418-420

ISSN : 0039-2944

\section{Référence électronique}

Elena Pessini, «Aa. Vv., Sexualité et écriture », Studi Francesi [En ligne], 143 (XLVIII | II) | 2004, mis en ligne le 30 novembre 2015, consulté le 19 mai 2021. URL : http://journals.openedition.org/ studifrancesi/39886 ; DOI : https://doi.org/10.4000/studifrancesi.39886

\section{Ce document a été généré automatiquement le 19 mai 2021}

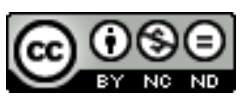

Studi Francesi è distribuita con Licenza Creative Commons Attribuzione - Non commerciale - Non opere derivate 4.0 Internazionale. 


\section{Aa. Vv., Sexualité et écriture}

\section{Elena Pessini}

\section{RÉFÉRENCE}

«Notre Librairie, Revue des littératures du Sud», Sexualité et écriture, n. 151, Juilletseptembre 2003, $143 \mathrm{pp}$.

1 Le numéro 151 de la revue "Notre Librairie" tient un pari qui relève du défi. L'avantpropos du directeur éditorial Jean-Louis Joubert souligne bien que consacrer un numéro entier à la thématique de la sexualité et à ses rapports avec l'écriture au sein des littératures du Sud est un signe des temps, un signe qui se veut porteur et témoin des évolutions certaines que l'on peut photographier tout au long du $\mathrm{XX}^{\mathrm{e}}$ siècle et à l'aube du nouveau millénaire.

2 Trois sections critiques composent la revue: "Écrire et décrire", "Les formes du discours", "Repères" et un quatrième volet contient des textes inédits. La première partie présente en ouverture un texte très précieux de Daniel Delas, "Décrire la relation: de l'implicite au cru", qui offre un panorama chronologique et critique des rapports entre sexualité et écriture dans les textes appartenant aux littératures africaines et antillaises. Des jalons fondamentaux y sont posés qui permettent de tracer la courbe d'une évolution au cours de laquelle ces littératures sont passées d'un silence pudique, d'une volontaire occultation des choses sexuelles où les écrivains pratiquent une auto-censure, à une phase intermédiaire où la sexualité commence à entrer dans les textes. C'est, selon Daniel Delas, la publication de la part de Yambo Ouologuem du Devoir de violence (1969) qui constitue le moment charnière de cette transformation. Sans pouvoir entrer dans le détail de chaque œuvre, le critique montre bien, par son analyse et à travers les ouvrages cités, que les littératures africaines et antillaises sont concernées par ce changement de cap. Pour cette seconde période sont indiquées non seulement des œuvres qui marquent, de par leur prise en compte de la relation et de la dimension sexuelles, de nouvelles tendances, mais loin d'être un simple inventaire, la réflexion de Daniel Delas offre aussi des pistes utiles pour des recherches à venir. En 
dernier lieu, les écritures contemporaines sont examinées et le critique souligne combien ces littératures suivent le mouvement général de la littérature mondiale "dans le sens d'une écriture plus violente, plus violemment sexualisée" (p. 14). Avec toutefois des particularités qui les distinguent, et notamment en ce qui concerne l'écriture féminine qui contribue à faire entrer dans les textes tout ce qui a trait à la sexualité, pour briser un long silence. La longue interview à Calixte Beyala L'écriture dans la peau qui clot la première partie répond en écho aux affirmations du critique et confirme cette prise en charge de la sexualité, jusqu'à ses extrêmes limites textuelles, de la part des écrivains femme. Dans son article "Entre désir et raison, le choix des comportements", Nathalie Carré applique sa solide réflexion aux littératures africaines et antillaises mais s'attache à des problématiques qui convoquent de façon plus ample les problématiques du désir et de la raison, de l'image, de la norme et de la pudeur en littérature. La contribution la plus surprenante, de par son objet, est celle que nous fournit Tanella Boni. Dans L'éducation sentimentale selon Adoras, l'auteur analyse un phénomène éditorial intéressant: la naissance au sein des Nouvelles Éditions Ivoiriennes d'une jeune collection proposant des titres (Premiers frissons, Un bonheur inattendu, Tendres confidences, Romances à l'île Boulay) qui invitent au rêve mais surtout qui s'affichent comme des histoires d'amour roses et sucrées. C'est sur l'énorme succès de ce "produit" que s'interroge Tanella Boni en examinant non seulement les histoires narrées mais surtout les stratégies qui sont mises en œuvre pour assurer une large diffusion. La clef des records du chiffre de vente ne tient pas tant à la qualité littéraires des textes mais plutôt à l'objectif qui a été fixé: fournir les ingrédients d'une "bonne" éducation sentimentale. Une éducation sentimentale que d'autres publications du même genre fournissent ailleurs (en Europe), selon les mêmes recettes. La conclusion péremptoire de Tanella Boni ouvre des perspectives sur les mesures qui nous permettront de juger les rapports entre les littératures "du Sud" et les autres: "Avec Adoras, la globalisation économique et la mondialisation culturelle ont de beaux jours devant elles, en Afrique. La collection, à mon sens, est un exemple patent du produit éditorial destiné à renflouer les caisses d'une entreprise qui, ainsi, passe allègrement le cap de toutes les crises sans les ressentir" (p. 31). Dans le dernier travail critique qui conclut cette première partie: L'écriture afro-brésilienne ou la poétique de l'érotisme, Nathalie Laval-Bourgade présente la poésie fortement sexualisée qu'abrite la revue "Cadernos Negros". À travers l'analyse des œuvres des poètes qui animent cette revue, et en particulier Sônia Fatima da Conceiçao, Regina Helena da Silva, Márcio Barbosa, Miriam Alves, Jamu Minka, le critique nous met face à des écritures qui refusent le filtre ou l'intermédiaire du symbole ou de la métaphore pour évoquer les sexes, l'acte sexuel, les manifestations physiques liées au désir, au plaisir et à leur assouvissement. Toutefois, cette plongée dans le cru de la chair nous montre bien comment ces textes n'ont pas la fin ultime de dire le sexe mais qu'ils parcourent la voie de la provocation pour lutter contre préjugés et stéréotypes.

3 La deuxième section interroge plus précisément la littérature à travers les trois genres qu'elle égrène: théâtre, roman et poésie. La première étude, présentée par Sylvie Chalaye, Des dramaturgies qui ont du corps ou la musicale culbute de la langue, a pour objet le théâtre africain francophone et s'interroge sur le long silence imposé au corps au cours de la période des Indépendances mais aussi dans les années 70 , lorsque le matériau des textes était davantage politique. Les œuvres théâtrales les plus récentes veulent combler cette lacune, ce grand vide, et les pièces se font "de chair et de sang". Les exemples donnés témoignent bien de cette entrée en scène du corps et l'éclairage 
critique invite à voir, au-delà des transgressions fortes, la nécessité de dire les violences subies au cours des siècles par le corps des Africains, sous le voile aussi d'une langue qu'il faut aujourd'hui "posséder" autrement. L'écriture romanesque est abordée en tant qu'écriture des femmes africaines et antillaises mais aussi chez deux écrivains congolais: Sony Labou Tansi et Henri Lopes. Le lecteur de ce numéro de "Notre Librairie" s'est déjà rendu compte, à la simple lecture de la première partie, que, dans la mise en texte de la sexualité, les femmes ont de plus en plus leur mot à dire. C'est ce que confirme l'étude d'Odile Cazenave Érotisme et sexualité dans le roman africain et antillais au féminin qui analyse, dans les deux aires géographiques (avec une petite incursion dans l'Océan Indien), les différentes modalités textuelles marquées par cette présence explicite ou implicite de la sexualité et/ou de l'érotisme. Boniface MongoMboussa, dans Deux approches de la sexualité dans le roman congolais, fait dialoguer les deux grands romanciers congolais et surtout leurs deux approches fort différentes de la sexualité à l'intérieur de leurs romans, approches qu'une expression lapidaire du critique nous semble parfaitement résumer: "En matière sexuelle, les personnages de Sony Labou Tansi sont des gourmands; ceux d'Henri Lopes des gourmets" (p. 67). Pour la poésie, la fine analyse de Babacar Sall: Poésie amoureuse d'Afrique noire et des diasporas interroge les rapports entre code amoureux et code poétique.

4 La dernière partie rassemble des contributions qui nourrissent la réflexion sous les angles de la psychanalyse, de la sociologie, de l'anthropologie et de la politique. Kangni Alem dans Le bestial au cœur de l'humain: imaginaire sexuel et représentations littéraires, sonde les replis des tabous sexuels, des limites à enfreindre, des "perversions" et traque leurs occurences ou leur absence dans les littératures africaines. Jacques Chevrier aborde la question du pouvoir lié à la sexualité. Dans Pouvoir, sexualité et subversion dans les littératures $d u$ Sud, il montre combien ces rapports, dans des sociétés qui furent colonisés, sont une vieille histoire que les écritures contemporaines veulent reparcourir. L'espace du roman, l'espace du texte, deviennent les lieux de sabotage du pouvoir par l'invention d'une langue explosive, pouvoir qui fut longtemps masculin et dont les écritures féminines, antillaises et africaines, ont le projet de se libérer. Les thèmes évoqués par Landry-Wilfrid Miampika dans L'autre et le semblable: la différence sont particulièrement intéressants à analyser au sein des littératures africaines qui rendent compte de sociétés qui se sont longtemps construites sur de fortes différences entre masculin et féminin, où le masculin sort vainqueur en particulier en termes de pouvoir. Cet article présente un excursus diachronique qui témoigne d'une évolution et s'interroge sur les nouvelles formes d'écriture qui tendent à nuancer les différences ou tout au moins è récupérer un féminin trop souvent occulté. On aurait pu s'attendre à lire davantage de travaux concernant les rapports entre la sexualité et le sacré en littérature. Seule Nathalie Schon travaille dans cette optique et sur un auteur en particulier. Dans Sexualité et sacré dans l'œuvre de Xavier Orville, elle effectue une lecture de toute l'œuvre de l'auteur antillais avec à la clef l'idée de la perte de la dimension du sacré dans le vécu sexuel qu'Orville dénonce à travers ses récits. Une vision "haute" de la sexualité qui est comparée à celle que nous offrent d'autres écrivains et en particulier Raphaël Confiant. Sous cet angle, Xavier Orville écrit en cavalier seul et son œuvre se situe comme une création fortement originale, loin des tendances les plus connues de la littérature antillaise.

5 La section des textes inédits enrichit et illustre les propos des critiques. Les trois genres littéraires sont représentés avec un extrait de la pièce Trans'haéliennes de Rodrigue Norman, un aperçu du roman Blue Bay, Palace de Nathacha Appanah-Mouriquand 
(encore à paraître), et une partie du poème Femme papyrus d'Anthony Phelps. Le très court passage d'un roman de la collection Adoras, Amour à vif, s'offre comme illustration des considérations critiques de Tanella Boni. Soulignons aussi que les nombreuses "Notes de lecture" qui présentent des ouvrages critiques et littéraires concernant le thème traité constituent un complément utile.

6 L'ensemble des travaux qui présente à la fois des analyses centrées de façon précise sur des auteurs et des réflexions qui convoquent un discours plus général sur les littératures du Sud, sur leurs rapports avec les autres littératures, constitue, nous semble-t-il, un outil précieux pour tout chercheur voulant explorer la thématique examinée. 